Molcoular biology Promen 18:143-147.1993

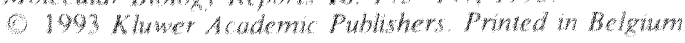

\title{
Structure and function of Signal Recognition Particle (SRP)
}

\author{
Henrich Lutcke \& Bernhard Dobberstein \\ Zentrum for Molekulare Bologie Heidelberg. Im Newenheiner fold 282, 69120 Heideberg. Gomany
}

Received and accepted 17 June 199 :

Key words: SRP. RNA-protein interaction

Signal recognition particle (SRP), a cytoplasmic ribonucleoprotein particle (for reviews see 135 , 191) serves as an adapter between ribosomes synthesizing secretory or membrane proteins and the translocation sites in the membrane of the rough endoplasmic reticulum (RER). SRP binds to the hydrophobic core of a signal sequence when it emerges from a ribosome. SRP then inhibits the further elongation of the nascent polypeptide, until it interacts with the docking protein (DP or SRP receptor), its receptor in the RER membrane [18]. The signal seguence is then released from SRP and is inserted into a proteinaceous translocation site in the RER membrane (for review, see [24]. SRP dissociates from its receptor and rejoins the pool of cytom plasmic SRP.

\section{Assembly of SRP}

The mammalian SRP is composed of $7 \mathrm{~S}$ RNA (or: SRP RNA) to which six polypeptides of 9 , $14,19,54,68$, and $72 \mathrm{kD}$ are attached either as monomers (SRP19 and SRP54) or as heterodimers (SRP9/14 and SRP68/72). SRP can

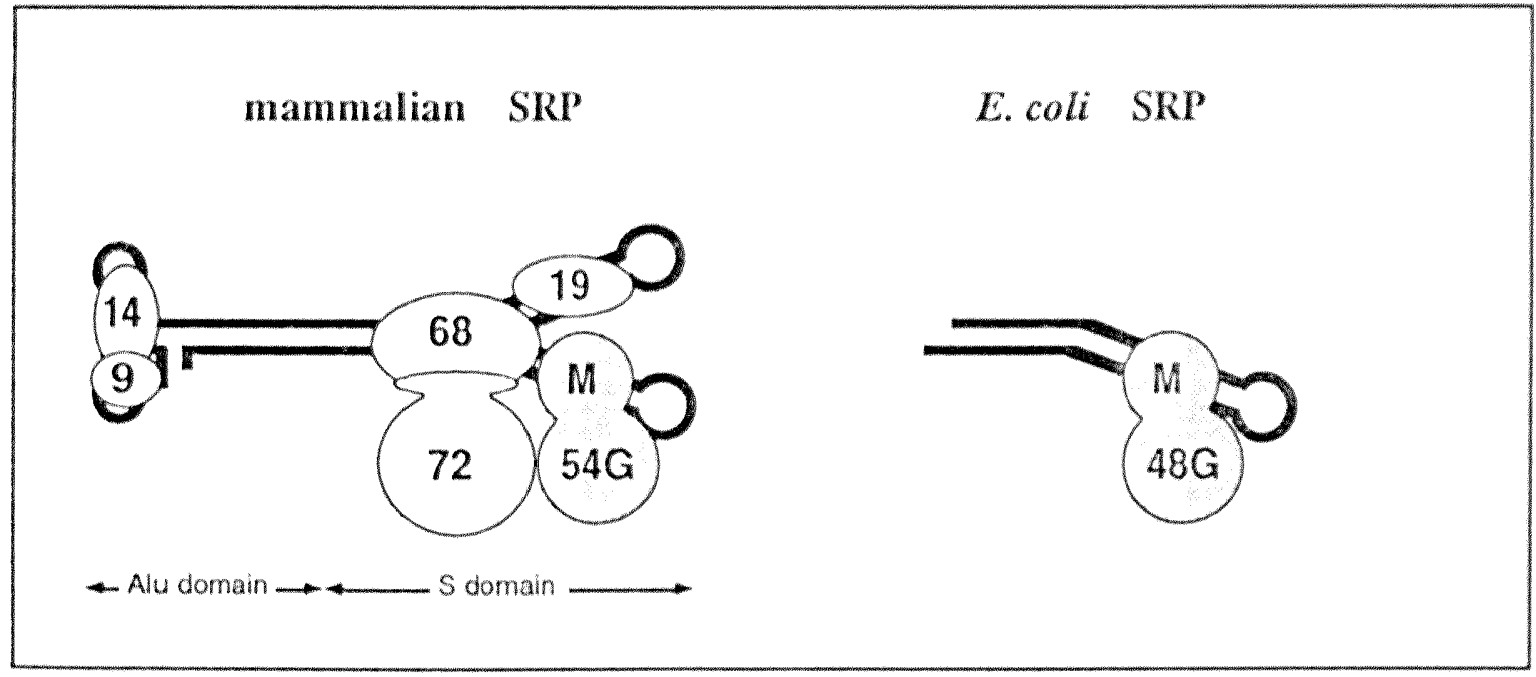

Fig. 1. SRP from mammalian and E. coll cells. The RNAs (7S RNA and 4.5S RNA, respectively) are depicted as thick lines. The protens are indicated by circles and identified by their molecular masses (kDa). For SRP54 and its E. coll homologue p48, the GTPase $(G)$ and the methonne-rich $(M)$ domains are indicated. 
be divided into two domains using nuclease (see Fig. 1). One domain (Alu domain) consists of the SRP9/14 heterodimer attached to the Alu sequences at the 5 and 3 ends of the 7 S RNA [7]. The other domain (S domain) consists of the remaining four largest proteins attached to the central so-called S fragment of the $7 \mathrm{~S}$ RNA (7).

Purfied SRP can be disassembled into its RNA and protein components and can be reconstituted from the isolated constituents into an active particle [34]. The isolated heterodimeric proteins. SRP9/14 and SRP68/72, cannot be separated into their respective polypeptide subunits under non-denaturing conditions. When the SRP proteins are expressed in vitro from their CDNAs and used to reconstitute SRP, they reveal different requirements for the assembly with $7 \mathrm{~S}$ RNA.

SRP9 and SRP14 associate with each other in the absence of 7S RNA and only subsequently can bind to 7S RNA [31]. Thus, the formation of an RNA binding site depends on the formation of the heterodimer.

In contrast, SRP68 binds to $7 \mathrm{~S}$ RNA in the absence of SRP72 via a highly positively charged region near the $\mathrm{N}$-terminus [17]. SRP72 only assembles into a complex with $7 \mathrm{~S}$ RNA in the presence of SRP68. The two in vitro synthesized proteins do not, or do very inefficiently, associate with each other in the absence of 7S RNA. C-terminal regions of both the SRP68 and the SRP72 are essential for the association of the two proteins with each other in the presence of $7 \mathrm{~S}$ RNA. Thus, SRP68 serves as an adapter for the assembly of SRP72 into a complex with the $7 \mathrm{~S}$ RNA [17; see Fig. 1)]

The two monomeric proteins, SRP19 and SRP54, bind directly to $7 \mathrm{~S}$ RNA $[14,27]$. SRP19 mediates the assembly of SRP54 with SRP RNA. This was found with the mammalian components in vitro [27] and with the homologous yeast components in vivo (in $S$. cerevisiae; [8]). No requirement for SRP19 has been found in the in vitro assembly of mammalian SRP54 with the smaller 7S RNA homologues of $E$. coli (4.5 S RNA) and B. subtilis (6S RNA) which lack the stem and loop of the 7S RNA to which SRP19 binds $[12,38]$.

\section{Signal sequence binding}

SRP54 is the signal sequence binding protein of SRP. It can be separated proteolytically into two fragments. The C-terminal fragment is highly positively charged and unusually rich in methionine residues and hence called $\mathrm{M}$ domain or SRP54M [1, 26]. SRP54M is necessary and sufficient for binding SRPS4 to the SRP RNA [27. 16]. The positive charges have been prow posed to line the hydrophilic faces of four amphipathic helices [1] and to mediate the binding to the SRP RNA [10].

The signal sequence binding site of SRP has also been mapped to SRP54M [37, 10]. SRP54M alone or reconstituted into SRP specifically interacts with a signal sequence exposed on a ribosome $[16,37]$, albeit with lower affinity than intact SRPS4 or SRP [37, Bacher and Dobberstein, unpublished observations]. A signal sequence binding pocket has been proposed to be formed by the hydrophobic faces of the amphipathic helices predicted to be present in SRP54M. The pocket would be lined by many methionine residues which, by virtue of their linear hydrophobic side chains, could readily adapt to the various signal sequences [1].

The proximity of the binding sites for signal sequences and for the SRP RNA within SRP54M suggests that the information about the binding of a signal sequence by SRP 54M may be relayed via the TS RNA to other regions of SRP. This in turn could effect subsequent activities like the inhibition of elongation.

The N-terminal domain of SRP54 (SRP54N + G or SRP54G) contains a predicted GTPase domain (similar to small GTPases) with an N-terminal extension $[1,26]$. SRP $54 N+G$ may be close to SRP54M since the alkylation of SRP54N+G with $N$-ethyl maleimide (NEM) prevents the binding of a signal sequence to SRP54M [16]. This, and the finding that the binding of a signal sequence to SRP $54 \mathrm{M}$ is inefficie gests th by whir require binding plexes. comple

The SF

Once : nascen the fur involve tution . alkylat unable less, si entire. the co. proteir intact

The inhibit the RI [20] at domai The in plex 0 interar

Docki

When nasce RER is rele Sec61 site [ brane (DP) dispo unit Muta 
ich

1 of

wo

thly

on-

or

and

NA

ro-

ohi-

$y$ to

has

10].

scif-

1 on

nity

and

sig-

ised

the

$t$ in

tany

heir

ıdily

gnal

thin

$\downarrow$ the

$y$ be

RP.

like

P54

cted

with

$+\mathrm{G}$

in of

EM)

e to

the

$A$ is inefficient in the absence of SRP $54 \mathrm{~N}+\mathrm{G}$, suggests that SRP54N+G may control the affinity by which SRP54M binds a signal sequence. No requirement for GTP has been detected for the binding of SRP to ribosome/nascent chain complexes. However, the release of SRP from such complexes requires GTP [2].

\section{The SRP-mediated pausing of elongation}

Once SRP has bound to a signal sequence in a nascent polypeptide, it can retard or even stop the further elongation [34]. This activity of SRP involves the Alu domain of SRP [28]. Reconstitution of SRP which omits SRP9/14, or includes alkylated SRP9/14, renders the resulting particles unable to inhibit the elongation [30]. Nevertheless, such particles, and even SRP lacking the entire Alu domain, are still capable of promoting the co-translational translocation of a secretory protein in vitro, albeit with lower efficiency than intact SRP [29].

The mechanism by which the elongation is inhibited is unknown. It has been proposed that the RNA in the Alu domain resembles a tRNA [20] and may thus exert its inhibitory effect in the domain of the ribosome to which tRNAs bind. The inhibition of elongation lasts until the complex of SRP, ribosome and nascent polypeptide interacts with the DP in the RER membrane [4]

\section{Docking of SRP to the RER membrane}

When SRP in the complex with ribosome and nascent chain interacts with the membrane of the RER in the presence of GTP, the signal sequence is released from SRP54 and makes contact with Sec61p, a protein component of the translocation site $[11,6]$. The component in the RER membrane to which SRP binds is the docking protein (DP) or SRP receptor.

DP is composed of a largely cytoplasmically disposed $\alpha$ - and a membrane-integrated $\beta$-subunit $[13,32]$ both of which bind GTP [2]. Mutational analysis of DP $\alpha$ suggests that GTP is bound by $\mathrm{DP} \alpha$ upon interacting with the complex of ribosome, nascent polypeptide and SRP and that this may trigger the release of the signal sequence from SRP as well as an increased affinity between SRP and DP [23]. Upon hydrolysis of GTP, the interaction between DP and SRP becomes sensitive to salt extraction [3].

Using reconstituted SRP, components have been identified which are necessary for mediating the targeting of a nascent secretory protein to microsomal membranes [30]. Selective alkylation of SRP68/72 in SRP allowed the modified SRP to still bind to a complex of ribosome and nascent polypeptide and to arrest the further elongation but prevented the interaction of the complex with the membrane [30]. Furthermore, the SRP68/72-alkylated SRP displayed a reduced affinity for DP. SRP lacking SRP54N $+\mathrm{G}$ likewise failed to promote targeting and to form a stable complex with DP [36]. Indeed, SRP54 appears to interact directly with DP, as a GTPase activity was greatly enhanced when both proteins were incubated in a purified system in the presence of SRP RNA [22]. Thus it appears that molecules required to mediate a stable interaction of SRP with the membrane, involve on the one hand SRP54 and SRP68/72, and on the other DP and possibly other membrane components.

\section{The release of the signal sequence from SRP}

Most recently, the reconstitution and functional characterization of membrane vesicles from detergent solubilized and fractionated microsomes has led to the identification of essential components of the translocation machinery in the RER membrane $[5,6]$. One such component essential for the translocation of model polypeptides was identified as the mammalian Sec61p [6]. This protein had previously been identified as the major RER membrane protein in contact with different signal sequences, once they had been released from SRP [11]. When membrane vesicles lacking sec61p were added to a translocation assay, the signal sequence remained bound to 
SRP54 as if no membranes had been added [D. Görlich and T. Rapoport. pers, communication]. The previously observed translocation defect of such vesicles may therefore be due to the failure of SRPS4 to release the signal sequence if no downstream acceptor for the signal sequence is available.

\section{SRP in other organisms}

Homologues of the SRP RNA have been described in eubacteria, archacbacteria, yeasts. plants, and mammals (for a compilation. see [12]), and particles homologous to SRP have been shown to function in $E$. coli $[21]$ and $S$. cerevisiae [9].

The SRP of E. coli contains the $48 \mathrm{kDa}$ homologue of SRP54 (p48 or Ffh) which is found in a complex with the $4.5 \mathrm{~S}$ RNA $[25,22$, cf. Fig. 1]. No other protein has been detected in the particle in addition to p48. P48 interacts with the signal sequence of nascent preprolactin in vitro [15] and, in E. coli, is essential for growth and the efficient export of some proteins [21]. The SRP of $S$. cerevisiae consists of one RNA molecule (scRl) and several proteins, one of which is the SRP54 homologue [9]. Like in E. coli, disruption of the SRP in S. cerevisiae differentially affects the secretion of different proteins. The disruption of the gene encoding SRP54 severely inhibits but does not completely abolish growth [9]. Thus, it appears that SRP is important for protein secretion in possibly all organisms.

\section{SRP, a signal sequence-specific chaperone}

SRP in its minimal form may be represented by the $E$. coli SRP. By binding to the hydrophobic core of a signal sequence it maintains it in a conformation competent for translocation. Thus, the minimal SRP can be considered a signal sequence-specific chaperone. Other chaperones (e.g. SecB, DnaJ/DnaK, GroEL/GroES, SecA) interact with the mature portion of secretory (pre)proteins and thereby may cooperate with
SRP in the post-translational translocation of proteins,

\section{References}

1. Bernsten HD. Porte M. Strub K. Hoben PI, Brenner, S \&. Walter P ( 1989 ) Nature $340: 482-486$

2. Connolly $\mathrm{T}$ \& Clmor R (1989) Coll $57: 599 \ldots 610$

3. Comnolly T. Rapiciko PJ \& Gimote R (1991) Science $252.1171-1173$

4. Gimore R \& Bbbel G (1983) Cell 35:677-w685

3. Gorlich D. Hatmant E. Prehn S k Rapopor TA (1992n) Nature $357: 47 \ldots 2$

6. Gorlich D. Prehn S. Martmann E, Katies K.U \& Rapow port $\mathrm{TA}(1992 b) \mathrm{Cell} 7 \mathrm{l}: 489-303$

7. Gundelfnger ED, Krauge E, Mell M \& Dobberstein B (1983) Nucletc Acids Res, 1 1: $7363-7374$

8. Harn BC. Stirling CJ Walter P (1902) Nature 356 $532-533$

9. Hann BC \& Walter P(1991) Cell 67: 131-144

10. High $S$ \& Dobberstein B (1991) I. Cell Biol. 113: $229-233$

11. High S, Gorlich D, Wiedmann M, Rapoport TA \& Dobberstein B (1991) J. Cell Biol. 113:35-44

12. Larsen $N$ \& Zwieb C (1991) Nucleic Acids Res. 19 $209-213$

13. Laufer L, Garcia PD, Harkins RN, Coussens L, Ullrich A \& Walter P (1985) Nature 318: $334-338$

14. Lingetbach K, Zwieb C, Webb JR, Marshalsay C, Hoben PJ, Walter P Dobberstein B (1988) Nucleic Acids Res. 16:9431-9442

15. Luirink 1, High $\$$, Wood H. Giner A, Tollervey D \& Dobberstein B (1992) Nature 359:741-743

16. Lutcke H, High S. Romisch K. Astford AJ \& Dobber $\operatorname{sicin}$ B (1992) EMBO J. 11: 1543-1551

17. Latcke H, Prehn S, Ashford AJ, Remus M, Frank R \& Dobberstein B (1993) J Cell Biol. 121:977-985

18. Meyer DI \& Dobberstein B (1980) J. Cell Biol 87: $503-508$

19. Nunnari J \& Watter p (1992) Curr. Op. Cell Biol. 4: $573-580$

20. Okada N (1990) J. Mol. Evol. 31:500-510

21. Phillips G. \& Silhavy TJ (1992) Nature 359: 744 746

22. Poritz MA, Bernstein HD, Strub K, Topf D, Wilhelm H \& Walter P (1990) Science 250: 1111-1117

23. Rapicjko PJ \& Gilmore R (1992) J. Cell Biol. 117 $493-503$

24. Rapoport TA (1992) Science 258:931-936

25. Ribes $V$, Romisch $K$, Giner A, Dobberstein B \& Tollervey D (1990) Cell 63:591-600

26. Romisch K, Webo J, Herz J, Prehn S, Frank R, Vingron $M \&$ Dobberstein B (1989) Nature 340:478-482

27. Romisch K. Webb J, Lingelbach K, Gausepohl H \& Dobberstein B (1990) J. Cell Biol. 111: 1793-1802 
3:

$\&$

9:

ch

C,

eic

\&

er-

\&

87:

4:

6

เ $\mathrm{H}$

17:

Tol-

ron

( \&
28. Siegel V \& Walter P (1985) J. Cell Biol. 100: 19131921

29. Siegel V \& Walter P (1986) Nature 320: 81-84

30. Siegel V \& Walter P (1988) Cell 52: 39-49

31. Strub K \& Walter P (1990) Mol. Cell. Biol. 10: 777784

32. Tajima S, Lauffer L, Rath VL \& Walter P (1986) J. Cell Biol. 103: 1167-1178
33. Walter P \& Blobel G (1981) J. Cell Biol. 91: 557-561

34. Walter P \& Blobel G (1983) Cell 34: 525-533

35. Walter P, Gilmore R \& Blobel G (1984) Cell 38: 5-8

36. Zopf D, Bernstein HD, Johnson AE \& Walter P (1990) EMBO J. 9: 4511-4517

37. Zopf D, Bernstein HD \& Walter P (1993) J. Cell Biol. 120: 1113-1121

38. Zwieb C (1991) Nucleic Acids Res. 19: 2955-2960 